\title{
Séminaires / Seminari 2014
}

\section{Ouverture et reprise d'un cabinet médical}

Le séminaire est destiné aux médecins sur le point d'ouvrir un cabinet médical (individuel ou de groupe), de joindre un cabinet de groupe ou de reprendre un cabinet existant.

\section{Contenu}

- Business plan (préparation du plan de financement et crédit d'exploitation, financement par la banque)

- Aménagement (implantation, projet et concept d'aménagement, choix du mobilier, budget)

- Estimation d'un cabinet (inventaire et goodwill)

- Laboratoire

- Administration d'un cabinet medical

- Assurances

- Passage du statut de salarié à celui d'indépendant

- Fiscalité

\section{Sponsors}

Les coûts sont pris en charge par divers sponsors (voir www.fmhservices.ch).

\begin{tabular}{lll}
$\begin{array}{l}\text { Dates } \\
\text { K20 }\end{array}$ & $\begin{array}{l}\text { Jeudi, 13 mars } 2014 \\
13.30-18.00 \mathrm{~h}\end{array}$ & $\begin{array}{l}\text { Lausanne World } \\
\text { Trade Center }\end{array}$ \\
\hline K21 & $\begin{array}{l}\text { Jeudi, } 8 \text { mai } 2014 \\
13.30-18.00 \mathrm{~h}\end{array}$ & $\begin{array}{l}\text { Genève } \\
\text { Crowne Plaza }\end{array}$
\end{tabular}

\section{Remise d'un cabinet médical}

Le séminaire s'adresse aux médecins désirant remettre un cabinet médical. Idéalement 5-10 ans avant la remise prévue (pour des questions de taxation et prévoyance).

\section{Contenu}

- Aspects juridiques (autour du contrat de remise/reprise)

- Estimation d'un cabinet (inventaire et goodwill)

- Assurances (prévoyance, assurances à l'intérieur et autour du cabinet)

- Conséquences fiscales d'une remise.

\section{Sponsors}

Les coûts sont pris en charge par divers sponsors (voir www.fmhservices.ch).

\begin{tabular}{|c|c|c|}
\hline \multicolumn{3}{|c|}{ Dates } \\
\hline K24 & $\begin{array}{l}\text { Jeudi } \\
5 \text { juin } 2014 \\
17.00-21.30 \mathrm{~h}\end{array}$ & $\begin{array}{l}\text { Lausanne } \\
\text { World Trade } \\
\text { Center }\end{array}$ \\
\hline K25 & $\begin{array}{l}\text { Jeudi } \\
13 \text { novembre } 2014 \\
17.00-21.30 \text { h }\end{array}$ & $\begin{array}{l}\text { Genève } \\
\text { Crowne Plaza }\end{array}$ \\
\hline
\end{tabular}

\section{Cabinet de groupe}

Le séminaire s'adresse aux médecins en formation voulant exercer leur future activité en cabinet de groupe, et aux libres praticiens qui souhaitent affilier leur cabinet individuel à un cabinet de groupe.

\section{Contenu}

- Stratégie (objectifs du cabinet de groupe; structure de l'offre de prestations)

- Entrepreneurs (composition de l'équipe; règles de conduite; participation financière et modèles de rémunération)

- Finances \& droit (assurances, prévoyance et patrimoine; forme juridique, finances et impôts)

- Lieu d'implantation \& immobilier (exigences locales; analyse de la situation concurrentielle; aménagement du cabinet et coûts)

- Ressources humaines (qualifications; lois, règlements et contrats)

- Direction \& organisation (structure et processus; tâches, responsabilités et compétences)

- Informatique \& administration (attentes en matière de système informatique pour le cabinet; évaluation)

- Rapport d'expérience (rapport de l'expérience d'un médecin, cofondateur d'un cabinet de groupe de la région).

\section{Sponsors}

Les coûts sont pris en charge par divers sponsors (voir www.fmhservices.ch).

\section{Date}

K85

$\begin{array}{ll}\text { Jeudi } & \text { Lausanne } \\ 25 \text { septembre } 2014 & \text { World Trade } \\ 13.30-18.00 \mathrm{~h} & \text { Center }\end{array}$

\section{Apertura e rilevamento di uno studio} medico

Il seminario è destinato ai medici in procinto di aprire o di rilevare uno studio medico.

\section{Contenuto}

- Business Plan (preparazione del piano di finanziamento e del credito d'esercizio, prestito bancario)

- Pianificazione (insediamento, progetto e pianificazione, scelta del mobilio, budget)

- Valutazione di uno studio medico (inventario e goodwill)
- Amministrazione di uno studio medico (interna allo studio, rapporti con la banca)

- Assicurazioni (tutte le assicurazioni necessarie interne ed esterne allo studio)

- Passaggio dallo stato di dipendente a quello di indipendente

- Fiscalità.

\section{Sponsor}

Diversi sponsor si fanno carico delle spese (si rimanda al sito www.fmhservices.ch).

\begin{tabular}{|c|c|c|}
\hline \multicolumn{3}{|c|}{ Date } \\
\hline K50 & $\begin{array}{l}\text { Giovedì } \\
10 \text { aprile } 2014 \\
\text { dalle } 14.00 \text { alle } 18.00\end{array}$ & $\begin{array}{l}\text { Chiasso } \\
\text { FMH Fiduciaria } \\
\text { Services }\end{array}$ \\
\hline K51 & $\begin{array}{l}\text { Giovedì } \\
23 \text { ottobre } 2014 \\
\text { dalle } 14.00 \text { alle } 18.00\end{array}$ & $\begin{array}{l}\text { Chiasso } \\
\text { FMH Fiduciaria } \\
\text { Services }\end{array}$ \\
\hline
\end{tabular}

\section{Inscription et information /}

Iscrizioni e informazioni

www.fmhservices.ch ou FMH Consulting Services, Cornelia Fuchs, Burghöhe 1, 6208 Oberkirch, tél. 04192500 77, fax 0419210586.

\section{Remarque / Osservazioni}

Les adresses des participants aux séminaires dont les coûts sont couverts en partie ou totalement par des sponsors sont communiquées aux sponsors concernés.

Gli indirizzi dei partecipanti ai seminari, i cui costi sono coperti in parte o completamente da degli sponsor, vengono comunicati agli sponsor interessati.

\section{Conditions d'annulation / Condizion d'annullamento}

Un montant est perçu pour une absence ou une annulation. Il est de:

Un importo verrà rimborsato in caso di assenza $o$ annullamento. Esso sarà di:

- 50 CHF par personne dans les 15 jours avant le début du séminaire / 50 CHF per persona entro i 15 giorni prima dell'inizio del seminario;

- 100 CHF par personne dans les 7 jours avant le début du séminaire / 100 CHF per persona entro i 7 giorni prima dell'inizio del seminario. 